\title{
Blood levels of pro-inflammatory and anti- inflammatory cytokines during an oral glucose tolerance test in patients with symptoms suggesting reactive hypoglycemia
}

\author{
W. Eik Filho ${ }^{1,2}$, S.S. Marcon ${ }^{2,3}$, T. Krupek ${ }^{4}$, I.T.S. Previdelli ${ }^{5,6}$, O.C.N. Pereira ${ }^{5,6}$ \\ M.A.R.C.P. Silva ${ }^{4}$ and R.B. Bazotte ${ }^{4}$ \\ ${ }^{1}$ Disciplina de Endocrinologia, Departamento de Medicina, Universidade Estadual de Maringá, Maringá, PR, Brasil \\ ${ }^{2}$ Programa de Pós Graduação em Ciências da Saúde, Centro de Ciências da Saúde, Universidade Estadual de Maringá \\ Maringá, PR, Brasil \\ ${ }^{3}$ Departamento de Enfermagem, Universidade Estadual de Maringá, Maringá, PR, Brasil \\ ${ }^{4}$ Departamento de Farmacologia e Terapêutica, Universidade Estadual de Maringá, Maringá, PR, Brasil \\ ${ }^{5}$ Departamento de Estatística, Universidade Estadual de Maringá, Maringá, PR, Brasi \\ ${ }^{6}$ Programa de Pós Graduação em Bioestatística, Universidade Estadual de Maringá, Maringá, PR, Brasil
}

\begin{abstract}
We evaluated the impact of postprandial glycemia on blood levels of pro-inflammatory and anti-inflammatory cytokines during an oral glucose tolerance test in non-diabetic patients with symptoms suggesting reactive hypoglycemia. Eleven patients with clinical symptoms suggesting reactive hypoglycemia received an oral glucose solution $(75 \mathrm{~g})$. Blood was collected at 0 (baseline), 30, 60, 120 and 180 min after glucose ingestion and the plasma concentrations of interferon- $\alpha$ (IFN- $\alpha$ ), interferon- $\gamma$ (IFN- $\gamma$ ), interleukin-1 receptor antagonist (IL-1RA), interleukin 2 (IL-2), interleukin-2 receptor (IL-2R), interleukin 4 (IL-4), interleukin 6 (IL-6), interleukin 8 (IL-8), interleukin 10 (IL-10), interleukin-12 (IL-12), interleukin 13 (IL-13), interleukin 15 (IL-15), interleukin 17 (IL-17), IFN- $\gamma$ inducible protein 10 (IP-10), monocyte chemotactic protein 1 (MCP1), monokine induced by IFN- $\gamma$

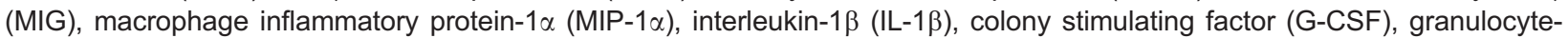
macrophage CSF (GM-CSF), basic fibroblast growth factor (FGF-basic), eotaxin, tumor necrosis factor $\alpha$ (TNF $\alpha$ ), epidermal growth factor (EGF), hepatocyte growth factor (HGF), vascular endothelial growth factor (VEGF), macrophage inflammatory protein-1 $\alpha$ (MIP-1 $\alpha$ ), and $1 \beta$ (MIP-1 $\beta$ ) were evaluated. Overall, glycemic levels increased, reached its maximum at 30 min (phase 1), returned to baseline levels at 120 min (phase 2), followed by a mild hypoglycemia at 180 min (phase 3). During phase 1, cytokine blood levels were maintained. However, we observed a synchronous fall $(P<0.05)$ in the concentrations of pro-inflammatory (IL-15, IL-17, MCP-1) and anti-inflammatory cytokines (FGF-basic, IL-13, IL-1RA) during phase 2. Furthermore, a simultaneous rise $(\mathrm{P}<0.05)$ of pro-inflammatory (IL-2, IL-5, IL-17) and anti-inflammatory cytokines (IL-4, IL1RA, IL-2R, IL-13, FGF-basic) occurred during phase 3. Thus, mild acute hypoglycemia but not a physiological increase of glycemia was associated with increased blood levels of anti-inflammatory and pro-inflammatory cytokines.
\end{abstract}

Key words: Pro-inflammatory cytokines; Anti-inflammatory cytokines; Glucose tolerance test; Inflammation; Postprandial glycemia

\section{Introduction}

Cytokines are small proteins with multiple biological properties (1-6) including growth factors (7), interferons (8), interleukins (9), chemokines (10) and the tumor necrosis factor family (11).

Early studies on cytokines focused on inflammation, but today it is recognized that cytokines mediate a multiplicity of biological processes. For example, interleukin 4, initially described as $\beta$ cell growth factor, also shows antiinflammatory properties, and leptin, initially described as a suppressor of appetite, also works as a pro-inflammatory cytokine (8).

On one hand, cytokines mediate a number of biological processes (8), and, on the other hand, a number of biological processes, including blood glucose changes $(12,13)$, influence 
cytokine blood levels. Moreover, in spite of the fact that acute hyperglycemia $(13)$ and hypoglycemia $(14,15)$ are associated with increased blood levels of pro-inflammatory cytokines in non-diabetic subjects, to our knowledge, there are no data that correlates anti-inflammatory cytokines with those conditions.

In this context, we aimed to evaluate the effect of the acute increase and decrease of glycemia on circulating proinflammatory and anti-inflammatory cytokines levels by assessing patients with clinical symptoms suggesting reactive hypoglycemia. The reason for selecting these specific individuals was the possibility to obtain a glycemic curve with a hyperglycemic followed by a hypoglycemic phase.

\section{Material and Methods}

The study was approved by the Ethics Committee of the Universidade Estadual de Maringá (Protocol CAAE \#0164.0.093.000-11) and written informed consent to participate in this investigation was obtained from each patient.

Patients seeking treatment for symptoms suggestive of reactive hypoglycemia at the first author's (W. E-F) private clinic (CLIPEN: Clínica e Pesquisa em Endocrinologia de Maringá, Paraná, Brazil) were recruited. Eligibility criteria were: age between 18 to 59 years, and agreement to participate in all of the activities proposed in the study. Exclusion criteria were: any type of diabetes, pregnancy or risk of pregnancy, breastfeeding, any disease or condition that could influence the investigation including cardiovascular, intestinal, liver, kidney and infectious diseases, body mass index $>30 \mathrm{~kg} / \mathrm{m}^{2}$, medical history of stroke, smoking, alcoholism or consumption of any illegal or medicinal drugs, elective surgery coinciding with the period of participation in the study, not understanding the study protocol, previous participation in other trials within 6 months prior to entry into this study.

A total of 11 patients were included in the study. On the day of the oral glucose tolerance test (OGTT), the patients (after 10-h overnight fast) received oral glucose solution (75 g), and venous blood samples were collected from the median cubital vein at 0 (baseline values), 30,60, 120, and $180 \mathrm{~min}$ after glucose ingestion. From the blood sample collected at time 0 , we measured glycated hemoglobin A1c, total cholesterol, triacylglycerol and C-peptide.

In addition, from plasma samples that were stored at $-80^{\circ} \mathrm{C}$, we measured plasma glucose and cytokines. ACTH, cortisol and insulin were also evaluated, as it is well established that their abnormal levels represent favorable conditions for hypoglycemia. Moreover, from the basal values (time 0 ) of glycemia and insulinemia, we calculated the homeostatic model assessment-estimated insulin resistance (HOMA-IR), according to the formula: fasting insulin $(\mu \mathrm{U} / \mathrm{L}) \times$ fasting glucose $(\mathrm{nmol} / \mathrm{L}) / 22.5$.

Hypoglycemia diagnosis during the OGTT was based on Whipple's triad, i.e., glycemia $<70 \mathrm{mg} / \mathrm{dL}$, symptoms consistent with hypoglycemia and improvement or resolution of symptomatology after elevation of glycemia.

Blood glucose, glycated hemoglobin A1c, total cholesterol and triacylglycerol were determined as previously described (16). Quantitative measurement of cortisol, ACTH, C-peptide and insulin were done using chemiluminescence enzyme immunoassay kits for use on the Immulite ${ }^{(B)}$ and Immulite ${ }^{\circledR} 1000$ systems (Siemens, USA).

We evaluated the following pro-inflammatory cytokines: interleukin $1 \beta$ (IL-1 $\beta$ ), interleukin 2 (IL-2), IL 2 receptor (IL-2R), interleukin 5 (IL-5), interleukin 6 (IL-6), interleukin 8 (IL-8), interleukin 12 (IL-12), interleukin 15 (IL-15), interleukin 17

Table 1. Individual characteristics of the 11 patients.

\begin{tabular}{lcccccccc}
\hline Patient & Age & Race & Gender & BMI & A/HC & GH A1c & HOMA-IR & Gly 180 \\
\hline 1 & 41 & Caucasian & F & 25.1 & 0.84 & 5.2 & 2.09 & 79.0 \\
2 & 26 & Caucasian & F & 18.3 & 0.82 & 4.1 & 0.86 & 52.0 \\
3 & 42 & Caucasian & F & 22.4 & 0.88 & 5.3 & 1.32 & 82.0 \\
4 & 45 & Caucasian & F & 27.8 & 0.88 & 4.2 & 3.40 & 97.0 \\
5 & 63 & Caucasian & F & 22.6 & 0.89 & 6.2 & 0.89 & 77.0 \\
6 & 33 & Caucasian & F & 23.9 & 0.81 & 5.8 & 2.65 & 83.0 \\
7 & 30 & Caucasian & F & 18.8 & 0.77 & 5.2 & 1.22 & 96.0 \\
8 & 51 & Caucasian & F & 22.0 & 0.89 & 5.0 & 0.93 & $47.0^{*}$ \\
9 & 49 & Caucasian & M & 22.0 & 0.92 & 5.3 & 0.73 & $63.0^{*}$ \\
10 & 58 & Caucasian & F & 28.7 & 0.91 & 6.0 & 1.26 & 90.0 \\
11 & 27 & Caucasian & F & 27.4 & 0.80 & 5.7 & 2.51 & 68.0 \\
\hline
\end{tabular}

Age: years; Gender: F (female), M (male); BMI (body mass index): $<18.5 \mathrm{~kg} / \mathrm{m}^{2}$ low weight, $18.5-24.9$ $\mathrm{kg} / \mathrm{m}^{2}$ normal weight, $25-29.9 \mathrm{~kg} / \mathrm{m}^{2}$ overweight; A/HC (abdominal/hip circumference): male normal $\leqslant 0.9 \mathrm{~cm}$, female normal: $\leqslant 0.85 \mathrm{~cm}$; GH A1c (glycated hemoglobin A1c) $<6.5 \%$; HOMA-IR (homeostatic model assessment-estimated insulin resistance). The normal values for HOMA-IR are based on BMI, i.e., BMI $<25 \mathrm{~kg} / \mathrm{m}^{2}$ : 0.4-2.9, BMI 25-30 kg/m²: 0.4-4.3, BMI >30 kg/m²: 0.7-8.2.; Gly 180: glycemic level $(\mathrm{mg} / \mathrm{dL})$ at $180 \mathrm{~min}$. ${ }^{*}$ Patients who showed signs of hypoglycemia. 
Table 2. Blood levels of evaluated parameters during $180 \mathrm{~min}$ after intake of a glucose solution $(75 \mathrm{~g})$.

\begin{tabular}{|c|c|c|c|c|c|}
\hline Substances & $0 \mathrm{~min}$ & $30 \mathrm{~min}$ & $60 \mathrm{~min}$ & $120 \min$ & $180 \min$ \\
\hline Glucose (mg/dL) & $90.9 \pm 3.5(11)$ & $135.1 \pm 9.9(11)$ & $115.7 \pm 7.6(11)$ & $94.9 \pm 4.3(11)$ & $75.8 \pm 5.0(11)$ \\
\hline Insulin $(\mu \mathrm{U} / \mathrm{mL})$ & $7.2 \pm 1.1(11)$ & $42.3 \pm 7.9(11)$ & $47.5 \pm 9.1(11)$ & $31.0 \pm 9.1(11)$ & $16.2 \pm 3.6(11)$ \\
\hline ACTH $(p g / m L)$ & $10.1 \pm 1.3(10)$ & $9.5 \pm 1.2(10)$ & $9.2 \pm 2.1(10)$ & $6.4 \pm 0.9(10)$ & $6.5 \pm 0.8(10)$ \\
\hline Cortisol ( $\mu \mathrm{g} / \mathrm{dL})$ & $17.0 \pm 2.1(10)$ & $15.3 \pm 2.3(10)$ & $14.5 \pm 2.2(10)$ & $10.8 \pm 1.6(10)$ & $8.4 \pm 1.5(10)$ \\
\hline IL-2 (pg/mL) & $7.0 \pm 1.6(7)$ & $7.5 \pm 2.8(11)$ & $8.4 \pm 4.9(9)$ & $4.1 \pm 1.4(7)$ & $8.6 \pm 2.1(9)$ \\
\hline IL-4 (pg/mL) & $15.5 \pm 3.7(9)$ & $23.6 \pm 8.8(11)$ & $23.7 \pm 13.6(10)$ & $8.5 \pm 1.3(10)$ & $23.4 \pm 5.9(10)$ \\
\hline IL-5 (pg/mL) & $3.0 \pm 0.8(8)$ & $5.5 \pm 2.0(9)$ & $6.0 \pm 4.1(7)$ & $2.6 \pm 1.1(6)$ & $5.0 \pm 1.4(9)$ \\
\hline IL-6 (pg/mL) & $4.3 \pm 1.7(10)$ & $5.1 \pm 1.8(11)$ & $5.4 \pm 2.2(10)$ & $4.8 \pm 2.4(9)$ & $5.8 \pm 2.4(11)$ \\
\hline IL-8 (pg/mL) & $14.9 \pm 1.8(10)$ & $17.7 \pm 3.3(11)$ & $17.9 \pm 4.8(11)$ & $12.7 \pm 1.0(11)$ & $16.3 \pm 2.1(11)$ \\
\hline IL-10 (pg/mL) & $12.4 \pm 5.8(11)$ & $13.9 \pm 6.4(11)$ & $14.2 \pm 6.3(11)$ & $11.9 \pm 5.5(11)$ & $7.8 \pm 3.2(11)$ \\
\hline IL-12 (pg/mL) & $99.5 \pm 6.3(11)$ & $104.8 \pm 7.1(11)$ & $105.9 \pm 8.9(11)$ & $97.5 \pm 4.1(11)$ & $108.6 \pm 5.3(11)$ \\
\hline IL-13 (pg/mL) & $36.9 \pm 5.5(9)$ & $44.4 \pm 7.9(11)$ & $43.6 \pm 12.2(9)$ & $24.9 \pm 3.5(10)$ & $41.1 \pm 6.8(10)$ \\
\hline IL-15 (pg/mL) & $54.8 \pm 12.6(11)$ & $62.2 \pm 19.0(10)$ & $64.1 \pm 33.6(9)$ & $29.1 \pm 7.1(10)$ & $28.5 \pm 8.1(11)$ \\
\hline IL-17 (pg/mL) & $10.0 \pm 3.2(7)$ & $10.9 \pm 4.8(7)$ & $18.1 \pm 14.8(4)$ & $1.3 \pm 0.4(6)$ & $4.7 \pm 1.5(4)$ \\
\hline $\mathrm{IL}-1 \beta(\mathrm{pg} / \mathrm{mL})$ & $28.1 \pm 7.6(10)$ & $35.0 \pm 11.0(11)$ & $34.4 \pm 13.5(11)$ & $20.3 \pm 4.6(11)$ & $30.3 \pm 6.7(11)$ \\
\hline IL-1RA (pg/mL) & $182.1 \pm 43.6$ & $204.5 \pm 59.9(8)$ & $212.4 \pm 105.7(6)$ & $80.5 \pm 32.9(4)$ & $217.3 \pm 41.6(7)$ \\
\hline IL-2R (pg/mL) & $134.5 \pm 28.6(10)$ & $201.6 \pm 65.9(11)$ & $215.1 \pm 111.7(10)$ & $84.6 \pm 12.5(10)$ & $193.4 \pm 48.5(11)$ \\
\hline $\mathrm{IFN}-\alpha(\mathrm{pg} / \mathrm{mL})$ & $25.3 \pm 2.6(10)$ & $30.5 \pm 5.5(11)$ & $27.8 \pm 7.8(11)$ & $19.3 \pm 1.2(11)$ & $29.8 \pm 4.3(11)$ \\
\hline $\mathrm{IFN}-\gamma(\mathrm{pg} / \mathrm{mL})$ & $20.8 \pm 1.8(10)$ & $24.5 \pm 4.7(11)$ & $24.4 \pm 6.5(11)$ & $17.6 \pm 1.0(11)$ & $24.7 \pm 3.2(11)$ \\
\hline MIG (pg/mL) & $57.0 \pm 9.2(11)$ & $69.6 \pm 12.8(10)$ & $64.7 \pm 16.4(10)$ & $45.5 \pm 6.0(10)$ & $50.7 \pm 6.8(10)$ \\
\hline TNF- $\alpha(p g / m L)$ & $4.8 \pm 1.9(8)$ & $33.1 \pm 26.8(10)$ & $30.7 \pm 24.0(8)$ & $168.3 \pm 167.0(9)$ & $2.7 \pm 0.9(6)$ \\
\hline $\mathrm{EGF}(\mathrm{pg} / \mathrm{mL})$ & $11.8 \pm 2.4(10)$ & $13.4 \pm 3.0(11)$ & $13.5 \pm 4.3(11)$ & $9.9 \pm 2.1(11)$ & $12.5 \pm 2.5(11)$ \\
\hline HGF (pg/mL) & $127.7 \pm 21.0(10)$ & $228.7 \pm 53.8(11)$ & $274.0 \pm 82.8(10)$ & $152.7 \pm 42.4(11)$ & $222.8 \pm 36.4(11)$ \\
\hline VEGF $(p g / m L)$ & $3.5 \pm 0.9(8)$ & $3.4 \pm 1.6(8)$ & $7.1 \pm 5.6(3)$ & $1.5 \pm 0.8(3)$ & $1.6 \pm 0.7(3)$ \\
\hline MIP-1 $\alpha(\mathrm{pg} / \mathrm{mL})$ & $30.4 \pm 3.5(11)$ & $30.7 \pm 5.2(11)$ & $31.0 \pm 7.9(10)$ & $22.3 \pm 1.0(11)$ & $23.1 \pm 1.5(11)$ \\
\hline MIP-1 $\beta(\mathrm{pg} / \mathrm{mL})$ & $43.0 \pm 6.4(11)$ & $44.5 \pm 10.1(11)$ & $44.7 \pm 15.7(10)$ & $29.0 \pm 3.1(11)$ & $29.2 \pm 3.4(11)$ \\
\hline G-CSF (pg/mL) & $115.9 \pm 16.3(10)$ & $158.8 \pm 38.2(11)$ & $152.8 \pm 63.1(11)$ & $84.1 \pm 5.8(11)$ & $144.4 \pm 26.5(11)$ \\
\hline GM-CSF (pg/mL) & $5.1 \pm 2.9(8)$ & $4.8 \pm 2.7(10)$ & $4.4 \pm 2.4(11)$ & $4.4 \pm 2.5(9)$ & $2.4 \pm 1.0(9)$ \\
\hline FGF Basic $(p g / m L)$ & $11.7 \pm 3.7(8)$ & $15.1 \pm 4.8(9)$ & $14.9 \pm 7.5(8)$ & $6.3 \pm 2.8(9)$ & $12.0 \pm 3.4(10)$ \\
\hline Eotaxin $(\mathrm{pg} / \mathrm{mL})$ & $30.5 \pm 5.0(11)$ & $27.9 \pm 1.9(11)$ & $28.4 \pm 2.1(11)$ & $28.4 \pm 2.4(11)$ & $31.2 \pm 1.8(11)$ \\
\hline MCP-1 (pg/mL) & $138.0 \pm 14.3(11)$ & $124.7 \pm 12.4(11)$ & $124.2 \pm 15.9(10)$ & $101.1 \pm 5.6(11)$ & $116.3 \pm 6.4(11)$ \\
\hline $\mathrm{IP}-10(\mathrm{pg} / \mathrm{mL})$ & $14.9 \pm 1.7(11)$ & $15.0 \pm 1.9(11)$ & $15.0 \pm 1.8(10)$ & $13.8 \pm 1.8(11)$ & $13.5 \pm 1.5(11)$ \\
\hline
\end{tabular}

Data are reported as means \pm SD with number of patients in parentheses. IL: interleukin; IL-1RA: IL-1 receptor antagonist; IL-2RI: L-2 receptor; IFN- $\alpha$ and IFN- $\gamma$ : interferon $\alpha$ and $\gamma$; MIG: monokine induced by IFN- $\gamma$; TNF $\alpha$ : tumor necrosis factor $\alpha$; EGF: epidermal growth factor; HGFh: epatocyte growth factor; VEGF: vascular endothelial growth factor; MIP-1 $\alpha$ and MIP-1 $\beta$ : macrophage inflammatory protein-1 $\alpha$ and $1 \beta$; G-CSF: granulocyte-colony stimulating factor; GM-CSF: granulocyte-macrophage stimulating factor; FGF-basic: basic fibroblast growth factor; MCP-1: eotaxin, monocyte chemotactic protein 1; IP-10: inducible protein 10.

(IL-17), epidermal growth factor (EGF), vascular endothelial growth factor (VEGF), eotaxin, granulocyte-macrophage colony-stimulating factor (GM-CSF), interferon $\alpha$ (IFN- $\alpha$ ), interferon $\gamma$ (IFN- $\gamma)$, monokine induced by IFN- $\gamma$ (MIG), inducible protein 10 (IP-10), monocyte chemotactic protein 1 (MCP-1), macrophage inflammatory protein-1 $\alpha$ (MIP-1- $\alpha$ ), macrophage inflammatory protein-1 $\beta$ (MIP-1- $\beta$ ), and tumor necrosis factor $\alpha(\mathrm{TNF}-\alpha)$.

Furthermore, we also evaluated the anti-inflammatory cytokines: interleukin 1 receptor antagonist (IL-1RA), interleukin 4 (IL-4), interleukin 6 (IL-6), interleukin 10 (IL-10), interleukin 13 (IL-13), basic fibroblast growth factor (FGF- basic), granulocyte-colony stimulating factor (G-CSF), and hepatocyte growth factor (HGF).

Cytokines were evaluated with the human cytokine magnetic plex panel (Invitrogen, USA) with the immunoassay Luminex Magpix ${ }^{\circledR}$ Plataform (USA).

A single database estimating a polynomial regression model of third degree to each variable response was obtained. Estimates generated from these adjustments were imputed into the database and the Hotteling $t$-test was used for paired comparisons between 0-30, 30-120, and 120-180 min. Results are reported as means \pm SD. $\mathrm{P}<0.05$ indicated statistical significance. 
Table 3. Variation of blood levels of cytokines during an oral glucose tolerance test.

\begin{tabular}{lccc}
\hline Parameters & $\begin{array}{c}\text { 0-30 min } \\
\text { (Phase 1) }\end{array}$ & $\begin{array}{c}\text { 30-120 min } \\
\text { (Phase 2) }\end{array}$ & $\begin{array}{c}\text { 120-180 min } \\
\text { (Phase 3) }\end{array}$ \\
\hline Glucose & $\uparrow$ & $\downarrow$ & $\downarrow$ \\
Insulin & $\uparrow$ & $\downarrow$ & \\
ACTH & $\downarrow$ & $\downarrow$ \\
Cortisol & & $\uparrow$ \\
Interleukine 2 & & $\uparrow$ \\
Interleukine 4 & & & $\uparrow$ \\
Interleukine 5 & & $\downarrow$ & $\uparrow$ \\
Interleukine 13 & & $\downarrow$ & $\uparrow$ \\
Interleukine 15 & & $\downarrow$ & $\uparrow$ \\
Interleukine 17 & & $\downarrow$ & $\uparrow$ \\
IL-1RA $^{\mathrm{b}}$ & & & $\uparrow$ \\
IL-2R $^{\mathrm{b}}$ & & $\downarrow$ & \\
VEGF-Basic $^{\mathrm{b}}$ & & $\downarrow$ & \\
MCP-1 $^{\mathrm{b}}$ & & & \\
\hline
\end{tabular}

The results were obtained by using the Hottelin test as described in Methods. $\uparrow$ : increase; $\downarrow$ : decrease; a: anti-inflammatory cytokine; b: pro-inflammatory cytokine; IL-1RA: IL-1 receptor antagonist; IL-2R: IL-2 receptor; VEGF: endothelial growth factor; MCP-1: monocyte chemotactic protein 1.

\section{Results}

The blood levels of glycated hemoglobin A1c, C-peptide, total cholesterol, and triacylglycerol were $5.3 \pm 0.2 \%(n=11)$, $1.9 \pm 0.2 \mathrm{mg} / \mathrm{mL}(\mathrm{n}=10), 189.0 \pm 10.1 \mathrm{mg} / \mathrm{dL} \quad(\mathrm{n}=11)$ and $119.4 \pm 4.2 \mathrm{mg} / \mathrm{dL}(\mathrm{n}=11)$, respectively.

In spite of the fact that all patients included in this study had clinical symptoms suggesting reactive hypoglycemia, during the OGTT only 2 patients showed glycemia $<70 \mathrm{mg} / \mathrm{dL}$ and other signs of hypoglycemia (Table 1). We observed a glycemic curve with 3 phases: a first phase characterized by an elevation $(P<0.05)$ of glycemia between 0 (baseline levels) and $30 \mathrm{~min}$ after glucose ingestion; a second phase characterized by a decrease $(P<0.05)$ of glycemia and return to baseline levels (30-120 $\mathrm{min})$, and a third phase (120-180 min) with an additional decrease of glycemia reaching values below $(P<0.05)$ baseline levels (Table 2).

During the OGTT, the blood insulin levels increased $(P<0.05)$ for 60 min after glucose ingestion and then progressively decreased until $180 \mathrm{~min}$, while blood levels of ACTH and cortisol progressively decreased between 0 and $180 \mathrm{~min}$. The HOMA-IR of all patients showed normal values (Table 1).

As shown in Table 3, during the first phase (0 to 30 min), blood levels of cytokines remained unchanged. In addition, part of the pro-inflammatory cytokines, i.e., IL-1 $\beta$, INF- $\alpha$, GM-CSF, EGF, VEGF, TNF- $\alpha$, MIG, IL-8, IP-10,
MIP-1- $\alpha$, eotaxin, IFN- $\gamma$, IL-12, MIP-1B, and the antiinflammatory cytokines, i.e., IL-10, G-CSF, and HGF remained unchanged during OGTT. Moreover, IL-6, categorized as both a pro-inflammatory and anti-inflammatory cytokine, also remained unchanged during OGTT. However, we observed a synchronous fall $(P<0.05)$ in the concentrations of pro-inflammatory (IL-15, IL-17, MCP-1) and anti-inflammatory cytokines (FGF-basic, IL-13, IL1RA) during phase 2. Furthermore, a simultaneous rise $(\mathrm{P}<0.05)$ of pro-inflammatory (IL-2, IL-5, IL-17) and antiinflammatory cytokines (IL-4, IL-1RA, IL-2R, IL-13, FGFbasic) occurred during phase 3 .

\section{Discussion}

Considering that the majority of previous studies focused on circulating pro-inflammatory cytokines, the evaluation of anti-inflammatory cytokines represents an advance to understand how blood glucose levels influence blood cytokines levels.

The decrease in blood levels of the pro-inflammatory cytokines seen in this study could be attributed, at least partly, to the anti-inflammatory effect of insulin (17) that showed increased blood levels for $120 \mathrm{~min}$ after glucose ingestion. On the other hand, the rise in the blood levels of the pro-inflammatory cytokines could be attributed, partly at least, to the progressive decrease of cortisol blood levels during the OGTT.

We suggest that the simultaneous fall (30-120 min) and rise (120-180 $\mathrm{min}$ ) of pro-inflammatory and antiinflammatory cytokines during OGTT is indicative of an initial anti-inflammatory and subsequent pro-inflammatory balance. This hypothesis is in agreement with previously reported studies showing toll like receptors agonists inducing both pro-inflammatory and anti-inflammatory cytokines (8). Furthermore, other studies also demonstrated activation of pro-inflammatory and anti-inflammatory cytokines during neonatal sepsis (18), systemic infections in preterm infants (19) and type 1 diabetes (20).

Our results showing a predominance of antiinflammatory effects associated with glycemia elevation in non-diabetic subjects contrasted with the results obtained by Esposito et al. (13). It must be considered, however, that those authors submitted their patients to non-physiological conditions including intravenous administration of glucose.

The novel findings of the present study were that mild acute hypoglycemia in non-diabetic subjects without insulin resistance (as inferred by HOMA-IR values) were associated with increased concentrations of pro-inflammatory and anti-inflammatory cytokines. This cytokine balance, as proposed by Elenkov et al. (6), could represent an important negative feedback mechanism, which protects the organism from an excessive inflammatory response and its consequences. 
Finally, our results indicated that not only pro-inflammatory cytokines but also anti-inflammatory cytokines must be evaluated to obtain a complete picture about the impact of glycemic changes on the circulating levels of cytokines.

\section{References}

1. Ribeiro AF, Nobre V, Neuenschwander LC, Teixeira AL, Xavier SG, Paula FD, et al. Use of inflammatory molecules to predict the occurrence of fever in onco-hematological patients with neutropenia. Braz J Med Biol Res 2013; 46: 200-206, doi: 10.1590/1414-431X20122397.

2. Tarantino G, Costantini S, Finelli C, Capone F, Guerriero E, La Sala N, et al. Is serum Interleukin-17 associated with early atherosclerosis in obese patients? J Transl Med 2014; 12: 214, doi: 10.1186/s12967-014-0214-1.

3. Boneberg EM, Hartung T. Molecular aspects of antiinflammatory action of G-CSF. Inflamm Res 2002; 51: 119-128, doi: 10.1007/PL00000283.

4. Opal SM, DePalo VA. Anti-inflammatory cytokines. Chest 2000; 117: 1162-1172, doi: 10.1378/chest.117.4.1162.

5. Zhang J, Middleton KK, Fu FH, Im HJ, Wang JH. HGF mediates the anti-inflammatory effects of PRP on injured tendons. PLoS One 2013; 8: e67303, doi: 10.1371/journal.pone.0067303.

6. Elenkov IJ, Chrousos GP. Stress hormones, proinflammatory and antiinflammatory cytokines, and autoimmunity. Ann N Y Acad Sci 2002; 966: 290-303, doi: 10.1111/j.17496632.2002.tb04229.x.

7. Alrouq FA, Al-Masri AA, Al-Dokhi LM, Alregaiey KA, Bayoumy NM, Zakareia FA. Study of the association of adrenomedullin and basic-fibroblast growth factors with the peripheral arterial blood flow and endothelial dysfunction biomarkers in type 2 diabetic patients with peripheral vascular insufficiency. J Biomed Sci 2014; 21: 94, doi: 10.1186/s12929-014-0094-y.

8. Dinarello CA. Historical insights into cytokines. Eur $J$ Immunol 2007; 37 (Suppl 1): S34-S45, doi: 10.1002/ eji.200737772.

9. Mahida YR, Gallagher A, Kurlak L, Hawkey CJ. Plasma and tissue interleukin-2 receptor levels in inflammatory bowel disease. Clin Exp Immunol 1990; 82: 75-80, doi: 10.1111/ j.1365-2249.1990.tb05406.x.

10. Ragusa F. Th1 chemokines in ulcerative colitis. Clin Ter 2015; 166: e126-e131, doi: 10.7417/T.2015.1835.

11. Zhang JM, An J. Cytokines, inflammation, and pain. Int Anesthesiol Clin 2007; 45: 27-37, doi: 10.1097/ AIA.0b013e318034194e.

\section{Acknowledgments}

This research was supported by PPSUS/Fundação Araucária - PR/SESA-PR/MS-Decit/CNPq.

12. Vinagre I, Sanchez-Quesada JL, Sanchez-Hernandez J, Santos D, Ordonez-Llanos J, de Leiva A, et al. Inflammatory biomarkers in type 2 diabetic patients: effect of glycemic control and impact of LDL subfraction phenotype. Cardiovasc Diabetol 2014; 13: 34, doi: 10.1186/1475-2840-13-34.

13. Esposito K, Nappo F, Marfella R, Giugliano G, Giugliano F, Ciotola $\mathrm{M}$, et al. Inflammatory cytokine concentrations are acutely increased by hyperglycemia in humans: role of oxidative stress. Circulation 2002; 106: 2067-2072, doi: 10.1161/01.CIR.0000034509.14906.AE.

14. Razavi Nematollahi L, Kitabchi AE, Stentz FB, Wan JY, Larijani BA, Tehrani MM, et al. Proinflammatory cytokines in response to insulin-induced hypoglycemic stress in healthy subjects. Metabolism 2009; 58: 443-448, doi: 10.1016/j.metabol.2008.10.018.

15. Ludwig MS, Minguetti-Camara VC, Heck TG, Scomazzon SP, Nunes PR, Bazotte RB, et al. Short-term but not longterm hypoglycaemia enhances plasma levels and hepatic expression of HSP72 in insulin-treated rats: an effect associated with increased IL- 6 levels but not with IL-10 or TNF-alpha. Mol Cell Biochem 2014; 397: 97-107, doi: 10.1007/s11010-014-2176-2.

16. Zubioli A, Batista MR, Stefanello TF, Carrara MA, Bazotte RB. Comparative performance of lab tests and blood testing device to monitor glucose, total cholesterol and triacylglycerol in type 2 diabetic patients. Braz J Pharm Sci 2011; 47: 615-621, doi: 10.1590/S1984-82502011000300020.

17. Sun Q, Li J, Gao F. New insights into insulin: The antiinflammatory effect and its clinical relevance. World $\mathrm{J}$ Diabetes 2014; 5: 89-96, doi: 10.4239/wjd.v5.i6.739.

18. Mancilla-Ramirez J, Ramirez-Herrera M, Portillo-Gomez L, Gaitan-Meza J, Dinarello CA. [Antagonists in neonatal sepsis]. Bol Med Hosp Infant Mex 1993; 50: 691-693.

19. Ng PC, Li K, Wong RPO, Chui K, Wong E, Li G, et al. Systemic infections in preterm infants. Arch Dis Child Fetal Neonatal Ed 2003; 88: F209-F213, doi: 10.1136/fn.88.3.F209.

20. Chatzigeorgiou A, Harokopos V, Mylona-Karagianni C, Tsouvalas E, Aidinis V, Kamper EF. The pattern of inflammatory/anti-inflammatory cytokines and chemokines in type 1 diabetic patients over time. Ann Med 2010; 42: 426-438, doi: 10.3109/07853890.2010.495951. 